\title{
UNIFORM ALGEBRAS AND PROJECTIONS
}

\author{
S. J. SIDNEY
}

\begin{abstract}
If $M$ is a closed $A$-submodule of $C(X)$ where $A$ is a uniform algebra on $X$ which contains a separating family of unimodular functions, and if $M$ is a quotient space of some $C(Y)$, then $M$ is an ideal in $C(X)$. If there is an example of a uniform algebra $A$ on some $X$ such that $A \neq C(X)$ but $A$ is complemented in $C(X)$, then there is such an example with $A$ separable.
\end{abstract}

1. Statements of results. The following problem was considered by Glicksberg [1]: If $A$ is a uniform algebra on $X$ which is (topologically linearly) complemented in $C(X)$, does it follow that $A=C(X)$ ? He obtained a number of positive results; subsequent work is summarized in Pełczyński's monograph [2].

We shall prove two results. The first reduces to the solution of a special case of Glicksberg's problem provided $M=A, Y=X$, and the given linear mapping is a projection (=idempotent linear transformation) on $C(X)$; the case $M=A$ can be readily deduced from Corollary 5.3 of [2]. The second result says that if the answer to Glicksberg's question is negative for some $A$, then it is negative for a separable $A$, a reduction which may prove useful in settling the problem. Recall that a uniform algebra on $X$ is a closed point-separating subalgebra (over $\mathbf{C}$ ) of $C(X)$ which contains the constant functions.

THEOREM 1. Let $A$ be a uniform algebra on $X$ which contains enough unimodular functions to separate the points of $X$, and let $M$ be a closed $A$-submodule of $C(X)$. If $M$ is the range of a continuous linear mapping from some $C(Y)$, then $M$ is an ideal in $C(X)$.

THEOREM 2. Suppose there is a uniform algebra $A$ on some $X$ such that $A \neq$ $C(X)$ but $A$ is complemented in $C(X)$. Then there is a separable uniform algebra $\tilde{A}$ on some $\tilde{X}$ such that $\tilde{A} \neq C(\tilde{X})$ but $\tilde{A}$ is complemented in $C(\tilde{X})$.

If $K$ is a nonempty closed subset of $X$ then the ideal $I=\{f \in C(X): f(x)=$ $0 \forall x \in K\}$ is necessarily complemented in $C(X)$ if $X$ is metrizable. However, if $X=\beta \mathbf{Z}$ the Stone-Cech compactification of the integers $\mathbf{Z}$ and if $K=X \backslash \mathbf{Z}$ then $I$ is not complemented in $C(X)$, that is, $c_{0}$ is not complemented in $l_{\infty}$; indeed, $c_{0}$ is not even a continuous linear image of $l_{\infty}$. Thus Theorem 1 has no obvious converse in the nonmetrizable case.

In Theorem 2, separability of $\tilde{A}$ is equivalent to metrizability of $\tilde{X}$. The proof will exhibit $\tilde{A}$ as a subalgebra of $A$ and $\tilde{X}$ as a quotient space of $X$; thus $\tilde{A}$ will be antisymmetric if $A$ is. It will be clear that the proof can be adapted to prove variants of the theorem in which, for example, complementedness is replaced by being a continuous linear image of some $C(Y)$.

Received by the editors June 23, 1983.

1980 Mathematics Subject Classification. Primary 46J10; Secondary 46E25.

Key words and phrases. Uniform algebra, projection, complemented.

(C) 1984 American Mathematical Society $0002-9939 / 84 \$ 1.00+\$ .25$ per page 
2. Proofs. The proof of Theorem 1 is modeled on that of [2, Proposition 4.1].

Proof of TheOREM 1. Let $K=\bigcap\left\{f^{-1}(0): f \in M\right\}, I=\{f \in C(X): f(x)=$ $0 \forall x \in K\}$. We must show that $M=I$. Suppose $M \neq I$. Then there is $\mu \in M^{\perp}$ not supported entirely in $K$. Thus there is $g_{0} \in M$ not vanishing identically on the support of $\mu$, so $0 \neq g_{0} \mu \in A^{\perp}$. Let $S$ denote the multiplicative semigroup of unimodular functions in $A$. By the Stone-Weierstrass theorem there are $u, v$ in $S$ such that $\left(g_{0} \mu\right)(u / v) \neq 0$. For $\nu \in M(X)$ (=regular complex Borel measures on $X)$ let $\nu^{*} \in M(T)\left(T\right.$ the unit circle in the complex plane) be $\nu^{*}(E)=\nu\left(v^{-1}(E)\right)$, that is, $\int_{T} f d \nu^{*}=\int_{X}(f \circ v) d \nu$ for $f \in C(T)$. For each $g \in M$,

$$
\int_{T} z^{n} d\left(g u v^{-1} \mu\right)^{*}=\int_{X} v^{n} d\left(g u v^{-1} \mu\right)=\int_{X}\left(g u v^{n-1}\right) d \mu=0
$$

for each positive integer $n$, so by the F. and M. Riesz theorem, $\Phi g=\left(g u v^{-1} \mu\right)^{*}$ lies in $H^{1} m$ where $m$ is Lebesgue measure on $T$ and $H^{1}$ is the usual Hardy space. Thus $\Phi$ maps $M$ into the separable dual $H^{1} m$ and is absolutely summing [2, Definition 0.2 . If $\Psi$ is a continuous linear mapping of $C(Y)$ onto $M$ then $\Phi \circ \Psi$ is absolutely summing, so compact [2, Theorem 0.5]; thus by the open mapping theorem $\Phi$ is compact, which in turn implies that $a \rightarrow \Phi\left((a \circ v) g_{0}\right)=a \Phi g_{0}$ is compact from the disc algebra to $H^{1} m$. This, however, is false: if $0 \neq h \in L^{1}(m)$ (the role of $h$ being played above by $\Phi g_{0}$ where $\left.\int_{T} d\left(\Phi g_{0}\right)=\int_{X} g_{0} u v^{-1} d \mu \neq 0\right)$ choose integers $N_{1}, N_{2}$ so that $\left|\int z^{N_{1}} h d m\right|=2 \varepsilon>0$ and (using the Riemann-Lebesgue lemma) $\left|\int z^{n} h d m\right|<\varepsilon$ whenever $|n| \geq N_{2}>\left|N_{1}\right|$; if $n_{1}, n_{2}$ are distinct (positive) integers then

$$
\begin{aligned}
\left\|z^{2 n_{1} N_{2}} h m-z^{2 n_{2} N_{2}} h m\right\|_{M(T)}=\left\|z^{N_{1}} h m-z^{2 n_{2} N_{2}+N_{1}-2 n_{1} N_{2}} h m\right\|_{M(T)} \\
\geq\left|\int z^{N_{1}} h d m\right|-\left|\int z^{N_{1}+2\left(n_{2}-n_{1}\right) N_{2}} h d m\right|>2 \varepsilon-\varepsilon=\varepsilon,
\end{aligned}
$$

contradicting the alleged compactness.

PROOF OF THEOREM 2. Let $P$ be a continuous projection on $C(X)$ with range $A$. We build increasing sequences $A_{0} \subset A_{1} \subset \cdots \subset A_{n} \subset \cdots$ and $B_{0} \subset B_{1} \subset \cdots \subset$ $B_{n} \subset \cdots$ of separable closed subalgebras of $C(X)$ with $B_{n}$ selfadjoint, $A_{n} \subset B_{n} \cap A$, and $P\left(B_{n}\right) \subset A_{n+1}$ as follows. Choose $h \in A$ such that $\bar{h} \notin A$. Let $A_{0}$ be the smallest closed subalgebra of $C(X)$ that contains $h$ and 1 . Then successively let $B_{n}$ be the closed selfadjoint subalgebra of $C(X)$ generated by $A_{n}$, and let $A_{n+1}$ be the closed subalgebra of $C(X)$ generated by $P\left(B_{n}\right)$. Let $A^{\prime}$ and $B^{\prime}$ denote the respective closures of $\bigcup A_{n}$ and $\bigcup B_{n}$. These are separable closed subalgebras of $C(X), B^{\prime}$ is selfadjoint while $A^{\prime}$ is not $\left(h \in A^{\prime} \subset A\right), A^{\prime} \subset B^{\prime}$ and $P\left(B^{\prime}\right)=A^{\prime}$. If $\tilde{X}$ is obtained from $X$ by collapsing each common set of constancy for $A_{\tilde{\tilde{X}}}^{\prime}$ (equivalently, for $\left.B^{\prime}\right)$ to a point, then $B^{\prime}$ and $A^{\prime}$ become the required $C(\tilde{X})$ and $\tilde{A}$.

\section{REFERENCES}

1. I. Glicksberg, Some uncomplemented function algebras, Trans. Amer. Math. Soc. 111 (1964), 121-137.

2. A. Pełczyński, Banach spaces of analytic functions and absolutely summing operators, CBMS Regional Conf. Ser. Math., No. 30, Amer. Math. Soc., Providence, R.I., 1977.

Department of MATHEMATICS, The University of CONNECTICUT, StorRs, ConNECTICUT 06268 